\title{
A Precis of Threshold Laws for Positron vs. Electron Impact Ionization of Atoms
}

\section{A. Temkin}

Heliophysics Science Division, NASA/Goddard Space Flight Center, Greenbelt, MD 20771, USA; aaron.temkin-1@nasa.gov

Received: 21 February 2020; Accepted: 1 April 2020; Published: 10 April 2020

check for updates

Abstract: The Coulomb-dipole theory of positron vs. electron impact ionization of hydrogen (as a proxy for neutral atoms) is reviewed, emphasizing how the analytic form of the threshold law (but not the magnitude) can be the same, whereas the physics of each is entirely different.

Keywords: Coulomb-dipole theory; positron vs. electron impact ionization

Threshold laws have been examined over a long period of time. It is not the purpose of this note to review the literature, but rather to concentrate on our own [1,2] approach and, in particular, to show how it applies to both positron as well as electron impact ionization, and to compare it with results from other Wannier [3] (electron) and modified Wannier (positron) approaches [4] to threshold laws for positron vs. electron impact ionization.

We start with the basic formula for the yield of two particles emerging from an initial state $\left(\Phi_{i}\right)$ :

$$
Q(E)=\iint\|M E\|^{2} \delta\left(E-\vec{k}_{1}^{2}-\vec{k}_{2}^{2}\right) d^{3} k_{1} d^{3} k_{2}
$$

where the transition matrix element is

$$
M E=\iint \Psi_{f}\left(\vec{k}_{1}, \vec{k}_{2}, \vec{r}_{1}, \vec{r}_{2}\right)\left[V_{t}\left(\vec{r}_{1}, \vec{r}_{2}\right)-V_{i}\left(\vec{r}_{1}, \vec{r}_{2}\right)\right] \Phi_{i}\left(\vec{r}_{1}, \vec{r}_{2}\right) d^{3} r_{1} d^{3} r_{2}
$$

The $M E$ is expressed in final state form, whereby $\Psi_{f}$ is, in principle, the exact solution of the Schrodinger equation, (potential $V_{t}$ ); $\Psi_{f}$ is normalized as a plane (s-partial) wave with unit amplitude at infinity, and $\Phi_{i}$ is the initial state before the interaction has taken place. Let us deal with electron/positron impact ionization of hydrogen. Then, the (s-wave) initial state is

$$
\Phi_{i}=\frac{\sin \left(k_{i} r_{1}\right)}{k_{i} r_{1}} \phi_{10}\left(r_{2}\right)
$$

Which implies (in Rydberg units)

$$
V_{i}=-\frac{2}{r_{2}}
$$

So that

$$
V t-V i=\mp \frac{2}{r_{1}} \pm \frac{2}{r_{12}}
$$

where the upper sign refers to electron and the lower sign positron scattering. Thus, the $M E$ becomes explicitly

$$
M E=\mp \iint \Psi_{f}^{\mp}\left(\vec{r}_{1}, \vec{r}_{2}, \vec{k}_{1}, \vec{k}_{2}\right)\left(\frac{2}{r_{1}}-\frac{2}{r_{12}}\right) \frac{\sin \left(k_{i} r_{1}\right)}{k_{i} r_{1}} \phi_{10}\left(r_{2}\right) d^{3} r_{1} d^{3} r_{2}
$$


This shows explicitly how the $M E$, from which the threshold law is derived, relates to the exact solution, $\Psi_{f}$, of the Schrodinger equation. The Wannier law [3] does not deal directly with the wave function, but is (in my opinion) a brilliant analysis of the final state treated as a classical system. For electron-atom (ion) ionization, it gives the well-known threshold law

$$
\left.Q_{-}{ }^{(W)}(E) \propto E^{-\frac{1}{4}+\frac{\mu}{2}}\right|_{\mu=\frac{1}{2}\left[\frac{100-9}{4 z-1}\right]^{\frac{1}{2}}} \propto E^{1.1268}
$$

where $\mathrm{z}=$ charge of the residual ion $(\mathrm{z}=1$ for neutral atom, specifically $H$, ionization). For positron impact, the classical theory is quite different, and Klar [4] has derived

$$
Q_{+}(E) \propto E^{2.65 \ldots}
$$

However, the classical assumption is not, in my opinion, fully justified. We believe the threshold is controlled by the region of phase space in which the outgoing particles do not share the available energy equally (i.e., the symbol $\triangleright$ means between a factor 2 and 10). In this region, the faster particle sees the slower particle plus the residual ion as a unit, which is a dipole, whose dipole moment is the distance of the slower particle from the residual ion. At the same time, the slower particle sees the residual ion directly, i.e., it is a pure Coulomb wave. Thus, the name Coulomb-dipole (CD) theory. Mathematically, this derives from the fact that the potential reduces as below in the CD region

$$
V_{t}=\mp \frac{2}{r_{1}}-\frac{2}{r_{2}} \pm \frac{2}{r_{12}} \cong-\frac{2}{r_{2}} \pm \frac{2 r_{2}}{r_{1}^{2}} \cos \left(\vartheta_{12}\right)
$$

For electron/positron impact, $\vartheta_{12}=\pi / 0$. This says in the electron case that the two outgoing electrons repel each other and come out on opposite sides of the residual ion (the proton in the case of hydrogen), but with one electron much farther out than the inner electron, such that it sees the inner electron and the residual ion as a dipole facing away from the faster, outgoing electron, whereas in the positron impact case the outgoing positron is the faster particle and it sees the dipole formed by the slower electron and the residual ion facing toward the faster particle. In both cases, the potential between the faster particle and the dipole is attractive; thus,

$$
V_{t}-V_{i} \cong-\frac{2 r_{2}}{r_{1}^{2}}
$$

Thus, the dipole moment seen by the faster particle is $=2 r_{2}$. At the same time, the slower particle (an electron in both cases) comes out seeing the full charge of the residual ion (as a pure Coulomb wave). The associated wave function is

$$
\Psi_{f} \propto F^{d}\left(r_{1}, r_{2}, k_{1}\right) F^{c}\left(r_{2}, k_{2}\right)
$$

This is so in both cases. Thus, the matrix element reduces to

$$
M E_{C D}\left(E, k_{1}, k_{2}\right) \propto \int_{0}^{\infty} r_{1}^{2} d r_{1} \int_{0}^{\frac{1}{2} r_{1}} r_{2}^{2} d r_{2} F^{d}\left(r_{1}, r_{2}, k_{1}\right) F^{c}\left(r_{2}, k_{2}\right)\left[\mp \frac{2}{r_{1}} \pm \frac{2}{r_{1} \pm r_{2}}\right] \frac{\sin \left(k_{i} r_{1}\right)}{k_{1} r_{1}} \phi_{10}\left(r_{2}\right)
$$

In (11), we have switched to the SSCL (Spherically Symmetric Contra/Co Linear) model [5], wherein the interaction $\frac{2}{r_{12}} \rightarrow \frac{2}{r_{1} \mp r_{2}}$, and in the CD region $\left(r_{1} \triangleright r_{2} ; k_{1} \triangleright k_{2}\right)$ the interaction in (11) 
reduces to $-\frac{2 r_{2}}{r_{1}{ }^{2}}$ (in the first approximation) in both cases (i.e., an attractive dipole). Thus, finally the $\mathrm{CD}$ matrix element reduces to

$$
M E_{C D}\left(E, k_{1}, k_{2}\right) \propto \int_{0}^{\infty} r_{1}^{2} d r_{1} \int_{0}^{\frac{1}{2} r_{1}} r_{2}^{2} d r_{2} F^{d}\left(r_{1}, r_{2} k_{1}\right) F^{c}\left(r_{2}, k_{2}\right)\left[-\frac{2 r_{2}}{r_{1}{ }^{2}}\right] \frac{\sin \left(k_{i} r_{1}\right)}{k_{1} r_{1}} \phi_{10}\left(r_{2}\right)
$$

From (12), it is clear that both the electron and positron threshold laws will have the same form. The derivation of the threshold law from (12) via Equation (1) is given in Ref. [1,2]; it is

$$
Q_{ \pm}(E) \propto \frac{E}{(\ln (E))^{2}}[1+C \sin (\alpha \ln (E)+\beta)]
$$

Note first that (13) is not a pure power law. However, it is important also to realize its limitations, which are contained in (12) where the proportionality constant and limits of integration imply:

(a) The constant of proportionality as between positron vs. electron impact ionization will be entirely different. (The respective Schrodinger equations are different. It is only in the respective CD regions that the wave functions have a similar form, cf. Equation (10); I would expect the electron to be much larger than the positron constant of proportionality.)

(b) The energy distribution cross section, from which (13) is derived does not include the nonCD region $k_{1} \geq k_{2} \geq \frac{1}{2} k_{1}$. This means a major part of the energy distribution (cross section) is not included. Specifically, when $\triangleright$ is interpreted $>2$, we find for $k_{2}{ }^{2}>(1 / 5) E$ that the energy distribution is not covered by the $\mathrm{CD}$ theory. The part that is covered has the following form [4]:

$$
\left.\sigma_{E}(\varepsilon) \propto \frac{[1+\cos (\alpha \ln (\varepsilon)+\beta))}{(\ln (\varepsilon))^{2}}\right]
$$

In (14), $\varepsilon$ is the energy of the slower electron in the region $\varepsilon<E / 5$. Nevertheless, the area under this restricted part of the energy distribution curve is, according to the CD theory [1], larger than the area under the middle part, which is the dominant contributor to the total yield curve in the threshold $\operatorname{limit}(E \rightarrow 0)[6,7]$.

We conclude by noting that a recent numerical calculation of the positron-atom impact cross section at very low energies by Bray et al. [8] gives results that are consistent with Klar's prediction. On the other hand, an experiment on positron-argon ionization [9], with an energy resolution not fine enough to test the modulation of the CD theory, found linear dependence, which is consistent with the $\mathrm{CD}$ theory $[1,2]$ in the first approximation.

Funding: This research received no external funding.

Conflicts of Interest: The authors declare no conflict of interest.

\section{References}

1. Temkin, A. Threshold Law for Electron-Atom Impact Ionization. Phys. Rev. Lett. 1982, 49, 365. [CrossRef]

2. Temkin, A.; Shertzer, J. Electron scattering from excited states of hydrogen: Implications for the ionization threshold law. Phys. Rev. A 2013, 87, 052718. [CrossRef]

3. Wannier, G.H. The threshold law for single ionization of atoms or ions by electron impact. Phys. Rev. 1953, 90, 817. [CrossRef]

4. Klar, H. Threshold ionization of atoms by positrons. J. Phys. B 1981, 14, 4165. [CrossRef]

5. Temkin, A.; Hahn, Y. Optical potential approach to the e- -atom impact ionization threshold problem. Phys. Rev. 1974, 10, 708. [CrossRef]

6. Temkin, A. The Coulomb-Dipole Theory of the $\mathrm{e}^{+/-}$-Atom Impact Ionization Threshold Law. In Electronic and Atomic Collisions; North-Holland: Amsterdam, The Netherlands, 1983; p. 755. 
7. Klar, H. Electronic and Atomic Collisions; North-Holland: Amsterdam, The Netherlands, 1984; p. 767.

8. Bray, I.; Bray, A.W.; Fursa, D.V.; Kadyrov, A.S. Near-Threshold Cross Sections for Electron qnd Positron Impact Ionization of Atomic Hydrogen. Phys. Rev. Lett. 2018, 121, 20341. [CrossRef]

9. Babij, T.J.; Machacek, J.R.; Murtagh, D.J.; Buckman, S.J.; Sullivan, J.P. Near thresholds ionization of argon by positrons. Phys. Rev. Lett. 2018, 120, 113401. [CrossRef] [PubMed]

(C) 2020 by the author. Licensee MDPI, Basel, Switzerland. This article is an open access article distributed under the terms and conditions of the Creative Commons Attribution (CC BY) license (http://creativecommons.org/licenses/by/4.0/). 